\title{
The usefulness of patient-specific 3D nasal silicone implant using 3D design and order form
}

\author{
Inhee Lee', Norihiro Ohba ${ }^{2}$, Hyejeong Lee ${ }^{3}$, Kyu-Sung Lee ${ }^{\mathrm{l}, *}$, Munjae Lee $\mathbb{D}^{4, *}$ \\ 'Department of Medical Device Management and Research, SAIHST, Sungkyunkwan University, Seoul, 06355, Republic of Korea; ${ }^{2}$ Primo Azabu-juban \\ Clinic, Minato Ku, Tokyo, Japan; ${ }^{3}$ The hills Aesthetic Surgical Clinic, Seoul, Republic of Korea; ${ }^{4}$ Department of Medical Humanities and Social Medicine, \\ Ajou University School of Medicine, Suwon, Gyeonggi-do, Republic of Korea \\ *These authors contributed equally to this work \\ Correspondence: Munjae Lee, Department of Medical Humanities and Social Medicine, Ajou University School of Medicine, Suwon, Gyeonggi-do, \\ Republic of Korea, Email emunjae@ajou.ac.kr
}

\begin{abstract}
Purpose: The need for customized implants has continuously increased, but patient-specific silicone implants are not yet commonly used in the plastic surgery market. We sought to validate the effectiveness of a 3D customized nasal implant design in terms of design and lead time compared with a manually customized implant by a surgeon.

Materials and Methods: Based on the computed tomography (CT) findings of 15 patients who planned rhinoplasty, a surgeon wrote order forms reflecting the surgical plan and subsequently designed implants manually using epoxy on a 3D printed skull. Separately, engineers analyzed the CT findings and designed 3D implants based on the order forms.

Results: Epoxy designs were 3D-scanned, converted into a stereolithography format and compared with 3D implant designs to assess which method had a smaller margin of error as per the preoperative order form. Moreover, the lead time in all steps are compared. Nasion thickness, tip thickness, glabella starting point, glabella width, radix width, and total volume were comparatively analyzed. In all parameters, the error rate of the 3D design is relatively lower than that of the epoxy design. The former also had a lower total volume and a faster manufacturing time.
\end{abstract}

Conclusion: With novel 3D customized nasal implants, the limitations of ready-made silicone implants are addressed, and it is now possible to preoperatively design implants more accurately, quickly, and conveniently.

Keywords: 3D customized silicone implant, rhinoplasty, silicone, prosthesis and implants, patient-specific implant

\section{Introduction}

Rhinoplasty is one of the most popular plastic surgeries. As Asians typically have a low nasal bridge, slight tip projection, and a short and wide nose, nasal augmentation has become very common. ${ }^{1-4}$ Rhinoplasty augmentation techniques have been developed over decades, and various kinds of materials have been used as a synthetic nasal implant, including silicone, expanded polytetrafluoroethylene (ePTFE), and autologous cartilages. ${ }^{1}$ Among them, silicone implants had been most frequently used in augmentation rhinoplasty in Asia. ${ }^{3}$ Silicone implants are inexpensive, convenient for carving to make a proper design, easily removable in case of side effects, and highly satisfactory in its design. ${ }^{5}$ However, silicone implants are only available in ready-made sizes. Trimming the bottom part to fit the nasal bone and upper lateral cartilage during surgery is necessary, and they require carving to alter the overall design and achieve good cosmetic outcomes. ${ }^{6}$ The trimming is manually performed by surgeons to meet the expectations of the patient as well as their own aesthetic standards; thus, repeated removal and insertion are necessary for modifications. ${ }^{7}$ However, it may not fit precisely, resulting in a gap between the bone and implant. Moreover, there is design limit with ready-made sizes. If patients need to cover glabella smoothly to compensate the lack of volume from forehead to nasion, it is almost impossible with ready-made as it cannot be carved with natural and thin., ${ }^{4,8}$ Therefore, several patients experience complications such as deviation, skin thinning, extrusion, and perforation and patient satisfaction is low since it is not possible to review the final implant design before surgery. ${ }^{3,9,10}$ However, as individuality and expression of personality have become more diverse and plastic surgery for self-satisfaction 


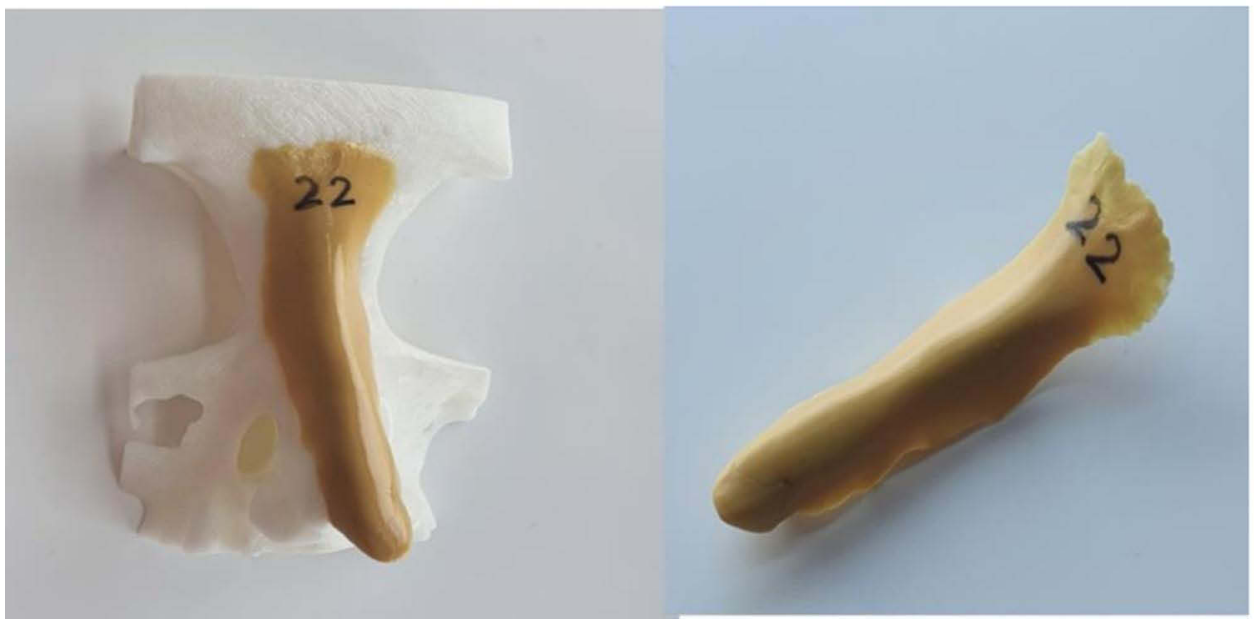

Figure I Epoxy-designed nasal implant based on a 3D skull.

has increased, the reflection of diversity and individuality in the design has become vital in achieving greater increase patient satisfaction. ${ }^{11}$ There was a resulting limit on simply trimming and using ready-made silicone implants with the advent of customized silicone implant manufacturing and medical 3D printing technology.

Currently, when a customized silicone implant is required for surgery, computed tomography (CT) scanning is used to print the patient's skull. The implant is manually designed using an epoxy rubber material, and the epoxy design is subsequently sent to a manufacturer for silicone implant production. ${ }^{12,13}$ This customized method received good feedback from surgeons, although it was complicated, labor-intensive, and, thus, difficult to apply on all patients.

In this study, we presented a new 3D customized manufacturing method to commercialize patient-specific silicone implants and analyzed the effectiveness of this design, which concerned most surgeons. Similar to other custom-made implants, surgeons fill out an order form, and engineers analyze the patient CT image, design implants based on order

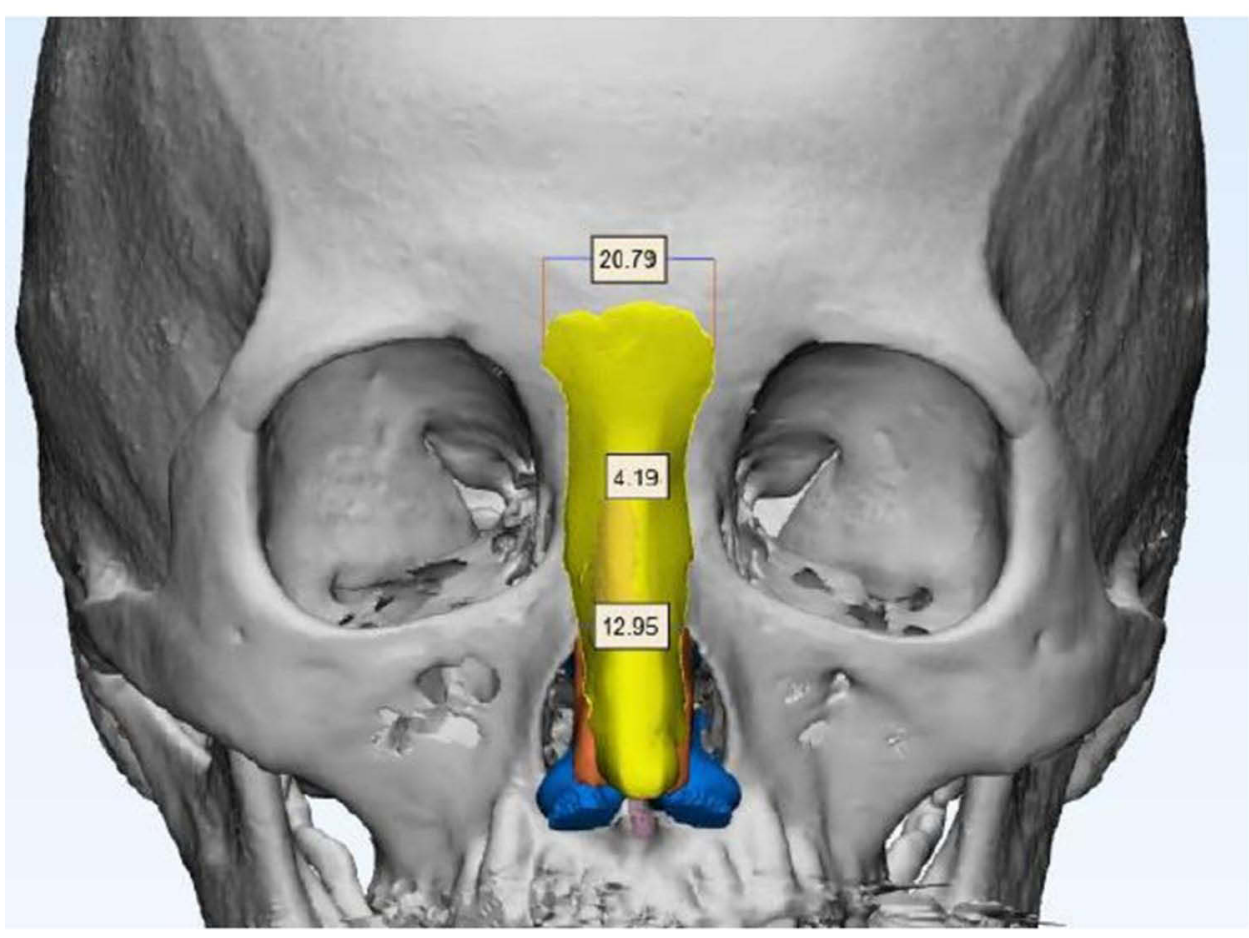

Figure 2 3D-scanned STL file of an epoxy design. 


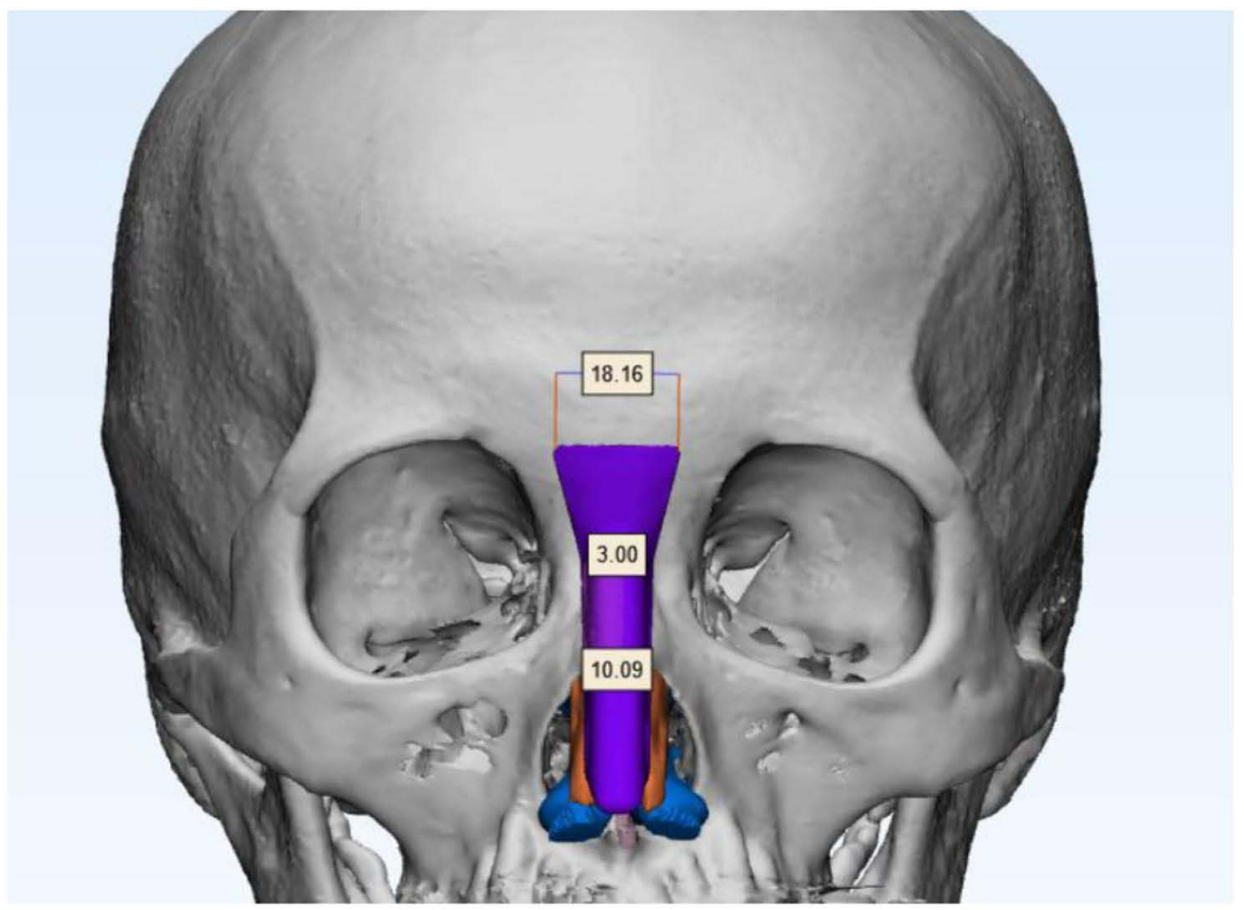

Figure 3 3D-designed nasal implant.
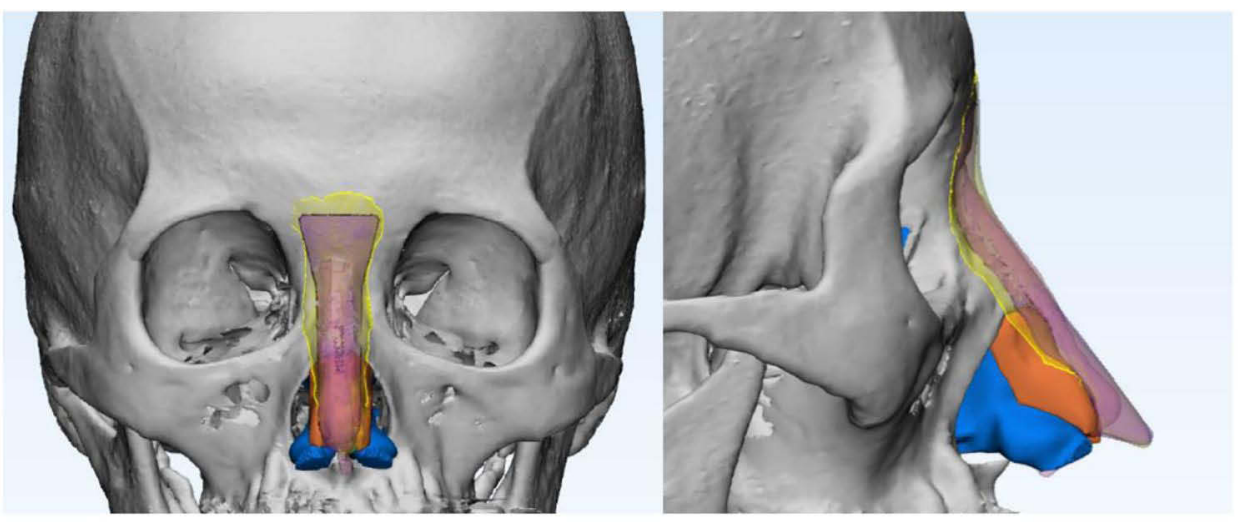

Figure 4 A side-by-side comparison of an epoxy design (yellow) and a 3D design (purple).

forms, and receive design confirmation from the requesting surgeon. We aimed to study the effectiveness of a 3D customized nasal implant. Unlike in general surgery, it is highly important and sensitive that the design components of the implant for rhinoplasty, such as nose line, thickness, and implant starting point, are finalized according to the surgeon's surgical plan and the patient's requests. ${ }^{8}$

\section{Materials and Methods}

\section{Overview of Study Design}

This study included 15 patients who underwent rhinoplasty at the Primo Azabu-juban Clinic, Japan between January 2018 and December 2020. All the patients had the main complaint of wanting to raise their nose or improve their undesirable status. At the same time as the nasal septal extension surgery, augmentation rhinoplasty was performed from glabella to supratip region. 
Design validation was performed using a four-stage experiment. In the first stage, all patients $(\mathrm{N}=15)$ underwent $\mathrm{CT}$ scanning and consultation with a surgeon, who filled out an order form reflecting the surgery plan and implant design. The order form provided by the FITme Co., Ltd. contained the criteria for selecting ready-made silicone implants for surgeons during rhinoplasty and the minimum condition for engineers to design implants at the FITme company.

In the second stage, the surgeon manually molded each nasal implant based on the corresponding order form using epoxy, PVA Clay (Tamiya, Shizuoka, Japan) on a 3D-printed skull (Figure 1). Each patient's CT findings were analyzed using Materialise Mimics software (Materialise, Leuven, Belgium) and 3D-printed by Poly-lactic Acid (PLA) using 3DWOXDP203 (Sindoh, Seoul, Korea). The epoxy material hardened a few hours later, and we 3D-scanned each epoxy design using Rexscan4 (Rapidscan3D, California, USA) and converted it into a standard 3D stereolithography (STL) file format (Figure 2).

In the third stage, 3D engineers from FITme Co., Ltd, Seoul, South Korea segmented patient CT images using Materialise Mimics ${ }^{14}$ and designed 3D patient-specific nasal implants using Materialize 3-matic (Materialise, Leuven, Belgium) according to order forms (Figure 3). ${ }^{15}$

In the fourth stage, we analyzed the error rate of the epoxy design and 3D design based on the order form standard, and all data in STL format were quantitatively analyzed (Figure 4). Between manufacturing methods, we compared the nasion and tip thickness, implant starting point, and its width, which the surgeon prioritized in rhinoplasty. Additionally, we analyzed the total volume of the two implants and lead time required for each stage.

\section{Order Form Criteria}

The main criteria are as follows:

1. Nasion thickness: Nasion is the midline bony depression between the eyes where the frontal and two nasal bones meet. It is also called the bridge of the nose, and the surgeon normally evaluates this for dorsum augmentation thickness.

2. Tip thickness: Tip thickness is determined by estimating how high the new nasal tip will be.

3. Implant starting point: 15 patients selected glabella as the starting point. Glabella is the most prominent point of the forehead, at the midpoint of the eyebrows.

4. Glabella width: This is the width of the implant starting point.

5. Radix width: This is equivalent to the implant width, and width point was set between the bone and cartilage.

6. Volume: This indicates the total volume of implants.

\section{Statistical Analysis}

The analysis of the collected data was performed using the SPSS25.0 program (IBM, New York, USA) and statistical significance was set at $\mathrm{p}<0.05$. The Wilcoxon signed-rank test was additionally performed to verify differences in the nasion and tip thickness, glabella starting point and width, radix width, total volume, and manufacturing lead time between two different manufacturing methods.

\section{Results}

\section{Descriptive Statistical Analysis of Variables}

Descriptive statistical analysis was performed to confirm the general trends of nasion thickness, tip thickness, glabella starting point, glabella width, radix width, total volume, and manufacturing time, which were the main variables of this study. The results are presented in Table 1. The 3D design had lower error rates in the nasion and tip thickness and at the glabella start point than the epoxy design. Additionally, it was confirmed that the 3D design had relatively lower error rates in glabella width and radix width, a relatively smaller volume, and a faster manufacturing time than the epoxy design. 
Table I Descriptive Statistics of Key Variables for Each Manufacturing Method ( $N=15)$

\begin{tabular}{|c|c|c|c|c|c|c|c|c|}
\hline \multirow[t]{2}{*}{ Variable } & \multicolumn{4}{|c|}{ Epoxy } & \multicolumn{4}{|c|}{ 3D Design } \\
\hline & $\mathbf{M}$ & SD & Min & Max & $\mathbf{M}$ & SD & Min & $\operatorname{Max}$ \\
\hline Nasion (mm) & 0.56 & 0.31 & 0.08 & 1.19 & 0.17 & 0.27 & 0.00 & 1.00 \\
\hline Tip (mm) & 1.98 & 1.08 & 0.05 & 3.94 & 1.33 & 0.93 & 0.06 & 2.90 \\
\hline Glabella starting point (error rate) & 2.96 & 2.17 & 0.40 & 7.56 & 1.24 & 0.91 & 0.40 & 3.99 \\
\hline Glabella width (mm) & 1.73 & 1.40 & 0.05 & 4.84 & 0.24 & 0.19 & 0.04 & 0.62 \\
\hline Radix width (mm) & 1.96 & 1.42 & 0.11 & 4.31 & 0.76 & 0.39 & 0.09 & 1.34 \\
\hline Total volume $(\mathrm{cm})$ & 24.62 & 5.25 & 13.45 & 31.48 & 17.78 & 4.14 & 11.73 & 25.56 \\
\hline Lead time (h) & 15.57 & 1.10 & 13.70 & 17.00 & 5.00 & 1.59 & 2.30 & 7.20 \\
\hline
\end{tabular}

\section{Differences in Key Variables by Manufacturing Method}

To verify the differences in nasion thickness, tip thickness, glabella starting point, glabella width, radix width, total volume, and lead time error range between the manufacturing methods, the Wilcoxon signed-ranks test was performed. The results are presented in Tables 2-6, and the analysis revealed significant differences in the error range between the two manufacturing methods, assessed using seven variables. Specifically, it was confirmed that the 3D designs had a smaller error range, shorter manufacturing time, and smaller size than the epoxy designs.

Table 2 Verification of Difference in Glabella Starting Point Error Rate Between Manufacturing Methods

\begin{tabular}{|l|c|c|c|c|}
\hline Method & $\mathbf{n}$ & Negative Ranks & Positive Ranks & $\mathbf{Z}(\boldsymbol{p})$ \\
\hline $\begin{array}{l}\text { Epoxy } \\
\text { 3D design }\end{array}$ & 15 & 108.00 & 12.00 & $-2.73^{* *}(0.006)$ \\
\hline
\end{tabular}

Note: $* * p<0.01$.

Table 3 Verification of Difference in Glabella Width Error Rate Between Manufacturing Methods

\begin{tabular}{|l|c|c|c|c|}
\hline Method & $\mathbf{n}$ & Negative Ranks & Positive Ranks & $\boldsymbol{Z}(\boldsymbol{p})$ \\
\hline $\begin{array}{l}\text { Epoxy } \\
\text { 3D design }\end{array}$ & 15 & 117.00 & 3.00 & $-3.24^{* *}(0.001)$ \\
\hline
\end{tabular}

Note: $* * p<0.01$.

Table 4 Validation of Difference in Radix Width Error Rate Between Manufacturing Methods

\begin{tabular}{|l|c|c|c|c|}
\hline Method & $\mathbf{n}$ & Negative Ranks & Positive Ranks & $\mathbf{Z}(\boldsymbol{p})$ \\
\hline $\begin{array}{l}\text { Epoxy } \\
\text { 3D design }\end{array}$ & 15 & 97.50 & 22.50 & $-2.13^{*}(0.033)$ \\
\hline
\end{tabular}

Note: $* p<0.05$.

Table 5 Verification of Difference in Total Volume Between Manufacturing Methods

\begin{tabular}{|l|c|c|c|c|}
\hline Method & $\mathbf{n}$ & Negative Ranks & Positive Ranks & $\mathbf{Z}(\boldsymbol{p})$ \\
\hline $\begin{array}{l}\text { Epoxy } \\
\text { 3D design }\end{array}$ & 15 & 120.00 & 0.00 & $-3.41^{* *}(0.001)$ \\
\hline
\end{tabular}

Note: ${ }^{* *} \mathrm{p}<0.01$. 
Table 6 Verification of Difference in Manufacturing Time Between Manufacturing Methods

\begin{tabular}{|l|l|c|c|c|}
\hline Method & $\mathbf{n}$ & Negative Ranks & Positive Ranks & $\boldsymbol{Z}(\boldsymbol{p})$ \\
\hline $\begin{array}{l}\text { epoxy } \\
\text { 3D design }\end{array}$ & 15 & 120.00 & 0.00 & $-3.41^{* *}(0.001)$ \\
\hline
\end{tabular}

Note: ${ }^{* *} \mathrm{p}<0.01$.

\section{Discussion}

Due to an increasing demand, various attempts have been made to develop customized implants optimized for rhinoplasty, which leads among performed operations in plastic surgery. ${ }^{2,10}$ In particular, the development of customized implants made of silicone, the most widely used material in plastic surgery, has received attention. Although ready-made silicone implants are inexpensive and readily available for surgery, ${ }^{5}$ these implants may not exactly fit the affected area; thus, displacement, bending, and gaps between implants, among others, are highly likely to occur. ${ }^{3,9,10}$ Moreover, as the implant design could not be confirmed prior to surgery, it affected postoperative patient satisfaction. ${ }^{11}$ In the customization of silicone implants, the patient's condition is accurately identified based on medical imaging, and the implant is designed to closely fit the affected area. The patient can also review the design of the implant before surgery. ${ }^{16}$ Specifically, it became possible to produce designs of the glabella area, which shows natural lines from the forehead to the nose and determines implant width and thickness. Such designs are suitable for individual facial structures and are not otherwise found in ready-made implants.

Previously, when implant customization was necessary, the surgeon would 3D print a skull with the patient's medical image and subsequently design an implant on it directly using rubber clay or epoxy. ${ }^{12}$ The designed epoxy would then be delivered to a silicone manufacturer, where a mold would be created using the material for dental plaster, and an implant would be produced with silicone. However, this method was not popularized due to the long 3D-printing time of the skull and epoxy design delivery period, as well as the burden on surgeons to design implants. Additionally, as it is important in plastic surgery to use an implant design that is appropriately based on patients' surgery purpose and surgeon's surgical plan, 3D implant designed by engineers has not been commercialized as in general surgery because of the effectiveness of the design. Therefore, in this study, a 3D design was found to be preferable for preoperative planning and was easier to order by converting both implant designs to STL files, which were compared by merging two datasets.

Expressly, a surgeon first sets the criteria for the surgical plan (A), then the surgeon designs an implant using epoxy on the skull (B) and engineers the 3D implants using a software based on an order form (C) filled by surgeons. The order form has certain conditions, including criteria that determine whether the surgeon should select a ready-made implant. The designs in $\mathrm{B}$ and $\mathrm{C}$ were then evaluated according to which better suits $\mathrm{A}$ and verified against seven variables to confirm manufacturing convenience. The variables were as follows: nasion thickness, tip thickness, glabella starting point, glabella width, radix width, total volume, and manufacturing time.

\section{Conclusions}

Results showed that custom-made silicone implants based on order forms are manufactured faster and more accurately than conventional silicone implants. A recent change in medical trends is the use of a customized treatment for each patient or the production of custom-made instruments to fit various individual body structures, shapes, or preference. In the production of customized devices, medical imaging and analysis, along with 3D printing, play a significant role. In rhinoplasty, which accounts for the largest proportion of surgeries in plastic surgery, the manufacturing of customized 3D nasal implants that have good commercial value in terms of design effectiveness, safety, delivery period, and cost is set to cause a large paradigm shift in the field of plastic surgery. It is likewise expected to expand commercially. 


\section{Data Sharing Statement}

Please contact the correspondence author for data availability.

\section{Institutional Review Board Statement}

This study protocol was approved by the Institutional Review Board of Samsung Hospital (IRB No.: SMC 2021-03-119). Since my advisor, professor Kyu-sung Lee is a professor at Samsung hospital, I was able to conduct IRB at Samsung hospital and the actual case study was processed at Primo Azabu-Juban clinic, Japan. Dr.Ohba who is a plastic surgeon and well versed in 3D Custom-made silicone implants collected patients suitable for the case and conducted the study. All data are kept anonymous. All methods were carried out in accordance with relevant guidelines and regulations. All patients provided informed consent to participate. The study was conducted in accordance with the Declaration of Helsinki.

\section{Author Contributions}

All authors made a significant contribution to the work reported, whether that is in the conception, study design, execution, acquisition of data, analysis and interpretation, or in all these areas; took part in drafting, revising or critically reviewing the article; gave final approval of the version to be published; have agreed on the journal to which the article has been submitted; and agree to be accountable for all aspects of the work.

\section{Funding}

We would like to thank the support of the Ministry of Education of the Republic of Korea and the National Research Foundation of Korea (NRF-2021R1I1A4A01057428) and Bio-convergence Technology Education Program through the Korea Institute for Advancement Technology (KIAT) funded by the Ministry of Trade, Industry and Energy (No. P0017805).

\section{Disclosure}

The authors declare no conflicts of interest for this work.

\section{References}

1. Bergeron L, Chen PKT. Asian rhinoplasty techniques. Semin Plast Surg. 2009;23(1):16-21. doi:10.1055/s-0028-1110097

2. Choi YD, Kim Y, Park E. Patient-specific augmentation rhinoplasty using a three-dimensional simulation program and three-dimensional printing. Aesthet Surg J. 2017;37(9):988-998. doi:10.1093/asj/sjx046

3. Khammoonta M, Kang K. Decreased complications due to application of aerocraft silicone implant in rhinoplasty of preselected patients. $J$ Cosmet Med. 2017;1(2):100-105. doi:10.25056/JCM.2017.1.2.100

4. Kim IS. Augmentation rhinoplasty using silicone implants. Facial Plast Surg Clin North Am. 2018;26(3):285-293. doi:10.1016/j.fsc.2018.03.003

5. Montri K, Kyongjin K. Decreased complications due to application of aerocraft silicone implant in rhinoplasty of preselected patients. $J$ Cosmet Med. 2018;2(1):100-105.

6. Lam SM, Kim Y-K. Augmentation rhinoplasty of the Asian nose with the "Bird" silicone implant. Ann Plast Surg. 2003;51(3):249-256. doi:10.1097/01.SAP.0000063756.49634.70

7. Yi H-G, Choi Y-J, Jung JW, et al. Three-dimensional printing of a patient-specific engineered nasal cartilage for augmentative rhinoplasty. $J$ Tissue Eng. 2019;10:2041731418824797. doi:10.1177/2041731418824797

8. Chang RH, Chang YL. Classification and treatment of glabella-radix deficiency in primary augmentation rhinoplasty. Aesthet Surg J Open Forum. 2020;2(2):ojaa016. doi:10.1093/asjof/ojaa016

9. Choi SM, Choi HJ, Kim CH, et al. Characteristics of nasal trauma in the implanted nasal prosthesis. J Korean Soc Plast Reconstr Surg. 2008;35 (5):597-602.

10. Han K, Kang J. A custom-made nasal implant: prefabrication from curing of silicone adhesive. Plast Reconstr Surg. 1996;97(2):436-444. doi:10.1097/00006534-199602000-00026

11. Pfaff MJ, Steinbacher DM. Plastic surgery applications using three-dimensional planning and computer-assisted design and manufacturing. Plast Reconstr Surg. 2016;137(3):603e-616e. doi:10.1097/01.prs.0000479970.22181.53

12. Yim HW, Nguyen A, Kim YK. Facial contouring surgery with custom silicone implants based on a 3D prototype model and ct-scan: a preliminary study. Aesthetic Plast Surg. 2015;39(3):418-424. doi:10.1007/s00266-015-0482-z

13. Kamali P, Dean D, Skoracki R, et al. The current role of three-dimensional printing in plastic surgery. Plast Reconstr Surg. 2016;137(3):1045-1055. doi:10.1097/01.prs.0000479977.37428.8e

14. Mao X, Li X, Jia J, et al. Validity and reliability of three-dimensional costal cartilage imaging for donor-site assessment and clinical application in microtia reconstruction patients: a prospective study of 22 cases. Clin Otolaryngol. 2020;45(2):204-210. doi:10.1111/coa.13491

15. Cho HR, Roh TS, Shim KW, et al. Skull reconstruction with custom made three-dimensional titanium implant. Arch Craniofac Surg. 2015;16 (1):11-16. doi:10.7181/acfs.2015.16.1.11 
16. Chavoin J-P, Grolleau J-L, Moreno B, et al. Correction of pectus excavatum by custom-made silicone implants: contribution of computer-aided design reconstruction. a 20-year experience and 401 cases. Plast Reconstr Surg. 2016;137(5):860e-871e. doi:10.1097/PRS.0000000000002071

\section{Publish your work in this journal}

Clinical, Cosmetic and Investigational Dermatology is an international, peer-reviewed, open access, online journal that focuses on the latest clinical and experimental research in all aspects of skin disease and cosmetic interventions. This journal is indexed on CAS. The manuscript management system is completely online and includes a very quick and fair peer-review system, which is all easy to use. Visit http://www. dovepress.com/testimonials.php to read real quotes from published authors.

Submit your manuscript here: https://www.dovepress.com/clinical-cosmetic-and-investigational-dermatology-journal 